\title{
Agonistas Dopaminérgicos no tratamento da Doença de Parkinson
}

\section{Dopamine Agonists on Parkinson's Disease treatment}

\author{
Henrique Ballalai Ferraz ${ }^{1}$
}

\begin{abstract}
RESUMO
A doença de Parkinson (DP) é a doença neurodegenerativa mais freqüente na população depois da doença de Alzheimer e é fonte de grave incapacitação para um grande número de pessoas. A doença é resultado de uma degeneração de neurônios da substância negra ocasionando uma deficiência de dopamina no corpo estriado. A reposição dopaminérgica com a levodopa, uma droga precursora da dopamina, é a pedra angular do tratamento da DP. Os agonistas dopaminérgicos (AD) são drogas que ativam diretamente os receptores de dopamina sem a necessidade de ser metabolizada pelo neurônio pré-sináptico. Há duas classes de $A D$, os ergolínicos e os não ergolínicos. Os derivados do ergot são a bromocriptina, pergolida, lisurida e cabergolina. Os não ergolínicos são a apomorfina, o pramipexol, ropinirol e o piribedil. O pramipexol e o ropinirol são mais bem tolerados que os demais. Embora menos potentes que a levodopa, os AD são eficazes na fase inicial da DP e permitem que se reduzam a incidência de complicações de longo prazo associados à levodopa, como as flutuações motoras e as discinesias. No tratamento da fase avançada da DP, são drogas úteis como coadjuvantes da levodopa minimizando as complicações. Há evidências recentes de que a utilização precoce dos $A D$, pramipexol e ropinirol, na DP poderia exercer um efeito neuroprotetor.
\end{abstract}

Unitermos: Doença de Parkinson, Agonistas dopaminérgicos, Levodopa, Neuroproteção.

\section{SUMMARY}

Parkinson's disease (PD) is one of the most frequent neurodegeneration and can induce severe disabling to some of the patients. Parkinsonian symptoms come out as a result of dopamine deficiency in basal ganglia due to neuron degeneration in the substantia nigra. Dopamine replacement with levodopa remains the mainstay despite the availability of new efficacious drugs to treat PD. Dopamine agonists (DA) directly activate dopamine receptors and do not require metabolic conversion by the presynaptic neuron. There are two different groups of DA: the ergoline derivates (bromocriptine, pergolide, lisuride, cabergoline) and the non-ergoline drugs (apomorphin, piribedil, ropinirole and pramipexole). The most recently launched DA, pramipexol and ropinirole, have a better tolerability profile as compared to the older agents. DA are effective on early phases of PD, although not in the same magnitude of levodopa. Early use of DA reduces the development of levodopa induced motor fluctuations and dyskinesias. Adding DA to levodopa on advanced phases of PD can minimize levodopa induced complications. There are evidences that early use of DA, particularly pramipexole and ropinirole, could exert some neuroprotective effect on PD patients.

Keywords: Parkinson's disease, Dopamine agonists, Levodopa, Neuroprotection.

Trabalho realizado: Setor de Distúrbios do Movimento da Disciplina de Neurologia da Universidade Federal de São Paulo

1 -Professor Afiliado e Chefe do Setor de Distúrbios do Movimento da Disciplina de Neurologia da Universidade Federal de São Paulo 


\section{INTRODUÇÃO}

A doença de Parkinson (DP) é, depois da doença de Alzheimer, a doença neurodegenerativa mais comum na população. Afeta indivíduos de ambos os sexos principalmente na faixa de idade acima de 50 anos, embora não seja tão incomum em pessoas mais jovens. É um transtorno motor que, se não tratado adequadamente, pode incapacitar seriamente o indivíduo acometido.

A doença foi descrita inicialmente por James Parkinson em 1817 e caracteriza-se por, na fase inicial, sintomas motores, como tremor de repouso nas extremidades e mento, rigidez muscular, acinesia e distúrbios do equilíbrio e da postura. Os sintomas costumam iniciar-se unilateralmente de forma lenta e gradual, tendendo a acometer os dois lados do corpo com o passar do tempo. É muito freqüente que, durante todo o tempo de evolução da doença, as manifestações clínicas permaneçam essencialmente assimétricas, sempre com um lado do corpo mais acometido que o outro.

$\mathrm{Na}$ fase mais avançada os distúrbios do equilíbrio e o acometimento da voz e da deglutição são freqüentes e fazem com que a qualidade de vida dos pacientes seja seriamente comprometida.

\section{Fisiopatologia da doença de Parkinson}

Do ponto de vista fisiopatológico ocorre uma redução na atividade dopaminérgica no nível do estriado. Isto ocorre porque há uma degeneração dos neurônios pigmentados da substância negra do mesencéfalo que se projetam para o corpo estriado, estrutura dos núcleos da base constituída pelo núcleo caudado e putâmen. Estes neurônios produzem dopamina que é liberada na sinapse. O neurônio pós-sináptico, por sua vez, projeta-se para o globo pálido, outra estrutura dos núcleos da base ${ }^{1}$. Este neurônio do estriado é do tipo espiculado ou espinhoso e de tamanho médio. Nele estão localizados os receptores dopaminérgicos. A razão porque ocorre a degeneração do neurônio dopaminérgico ainda permanece desconhecida, mas o tratamento dos sintomas da doença pode ser, ao menos parcialmente, bem sucedido se fizermos a restauração da atividade dopaminérgica estriatal.

Dizemos que o sucesso no tratamento sintomático da DP é parcialmente bem sucedido porque muitas das manifestações clínicas têm origem não dopaminérgica. Podemos citar as alterações de postura e equilíbrio, a disartrofonia, os bloqueios motores ("freezings"), entre outros que são pouco responsivos à reposição dopaminérgica. Há certamente outras vias neurais envolvidas na doença, como a noradrenérgica (pela degeneração do locus ceruleus), a serotonérgica (degeneração dos núcleos da rafe) e colinérgica (degeneração do núcleo basal de Meynert). Na verdade, é possível que a maior parte das vias degeneradas na DP ainda não seja completamente conhecida ${ }^{1}$.

O neurônio chave para entendermos a razão do sucesso e do insucesso da terapêutica de reposição dopaminérgica na DP é o neurônio espinhoso médio do estriado. Nele é que estão os receptores da dopamina. Há duas famílias de receptores dopaminérgicos: a família D1, diretamente ligada à adenil-ciclase e, portanto estimulatória e a família D2, ligada à proteína G e inibindo a adenil-ciclase, dessa forma diminuindo os níveis de AMP-cíclico e com ação inibitória. A ativação dopaminérgica no estriado promove simultaneamente a ativação da chamada via direta do circuito extrapiramidal, através dos receptores do tipo D1 e a inibição da via indireta através da estimulação dos receptores do tipo D2. Cada família de receptores, por sua vez, tem seus subtipos. A família D1 pode ser subdividida em receptores D1 e D5, enquanto que a família D2 em receptores D2, D3 e D4 ${ }^{2}$.

No neurônio espinhoso médio, existem outros receptores importantes para o tratamento da sintomatologia parkinsoniana. Neste neurônio há também receptores para o neurotransmissor glutamato, como os receptores do tipo N-metil-Daspartato (NMDA) e os receptores adenosina D2A. A estimulação ou o bloqueio destes receptores também parece ter um papel fundamental no tratamento da DP ${ }^{2}$.

\section{Drogas utilizadas no tratamento da doença de Parkinson}

Até os anos 1960 o tratamento da DP era baseado no uso de medicamentos com ação anticolinérgica e no tratamento cirúrgico, através da cirurgia ablativa de alvos localizados nos núcleos da base. O surgimento da levodopa fez revolucionar o tratamento ${ }^{3}$. A droga, precursora da dopamina, fez com que pacientes gravemente incapacitados voltassem a ter uma vida muito próxima do normal. A levodopa até hoje é a pedra angular do tratamento da DP. A levodopa é absorvida no duodeno e transportada pela corrente 
sangüínea até o cérebro onde penetra e é convertida em dopamina pela ação da enzima dopa descarboxilase (DDC). Esta conversão pode ocorrer nos neurônios dopaminérgicos remanescentes na substância negra ou por neurônios não dopaminérgicos ou ainda por células da glia na região do estriado. A meia vida plasmática da levodopa é muito curta, não passando de 90 minutos. Na corrente sangüínea a levodopa, antes de atingir o cérebro, é atacada por duas enzimas, a DDC e a Catecol-Orto-MetilTransferase (COMT). A DDC converte a levodopa em dopamina no sangue periférico, gerando manifestações dopaminérgicas periféricas, como náuseas, vômitos e hipotensão ortostática. A COMT converte a levodopa em 3-O-metil-dopa. Nas apresentações comerciais de levodopa sempre há a combinação com drogas inibidoras da ação periférica da DDC, como a benzerasida e a carbidopa. Há também drogas com ação inibitória sobre a COMT, o que faz aumentar o rendimento da levodopa, ou seja, aumentar a entrada de levodopa no cérebro. Há dois inibidores da COMT, o tolcapone e entacapone, este último também oferecido em comprimidos combinados à levodopa e à carbidopa.

Outras drogas utilizadas no tratamento da DP são a selegilina, a amantadina e os agonistas dopaminérgicos. A selegilina tem uma ação inibidora da enzima monoamino-oxidade (MAO) que uma das enzimas responsáveis pelo metabolismo intraneuronal da dopamina. Além disso, a selegilina tem uma ação semelhante à da anfetamina, fazendo uma estimulação catecolaminérgica. A amantadina tem um mecanismo de ação não completamente esclarecido, mas parece que sua ação antagonista de receptores NMDA é importante para sua ação antiparkinsoniana.

\section{Agonistas dopaminérgicos}

Os agonistas dopaminérgicos (AD) exercem seu efeito através da estimulação dos receptores dopaminérgicos localizados no neurônio pós sináptico. Por terem essa ação direta sobre o receptor não necessitam ser metabolizados previamente para atuarem como a levodopa. Há fortes evidências de que a ativação do receptor do tipo D2 é crucial para o efeito motor dos $A D$, mas a estimulação simultânea D1 e D2 parece ser necessária para que produza efeitos ótimos do ponto de vista fisiológico e comportamental ${ }^{4}$.
Os AD administrados por via oral têm uma meia vida que varia de 6 a 96 horas dependendo da droga e, por terem uma meia vida mais longa que a levodopa, produzem uma estimulação mais fisiológica dos receptores dopaminérgicos do que esta. Há agonistas que podem ser administrados por via parenteral e estes costumam também ter uma meia vida curta, como a levodopa.

A Tabela 1 mostra as duas classes de AD disponíveis atualmente para uso clínico na DP.

\begin{tabular}{|l|l|}
\hline Ergolínicos & Não ergolínicos \\
\hline Bromocriptina & Apomorfina \\
\hline Lisurida & Pramipexol \\
\hline Pergolida & Ropinirol \\
\hline Cabergolina & Piribedil \\
\hline
\end{tabular}

Tabela 1. Classes de agonistas dopaminérgicos.

\section{Agonistas ergolínicos}

São drogas derivadas dos alcalóides do ergot, tendo uma ação direta sobre os receptores dopaminérgicos. $O$ primeiro dos $A D$ utilizado na DP foi a bromocriptinsa em $1974^{5}$. A bromocriptina tem uma ação agonista sobre receptores D1 e antagonista sobre receptores D1. Tem uma meia vida de 6 horas e é muito pouco tolerada se tentarmos iniciá-la em doses maiores. Temos que iniciar com 1,25 mg à noite ao deitar e fazer incrementos semanais de 1,25 mg até conseguirmos atingir a dose mínima efetiva que é de cerca de 7,5 mg ao dia. Normalmente, demora-se de 6 a 8 semanas para que se consiga atingir essa dose. São prescritas, de um modo geral, três tomadas diárias da medicação. Os principais efeitos colaterais são as náuseas, vômitos e hipotensão postural que podem ser percebidas logo na primeira tomada. Também podem ser observados sonolência, tontura e quadros alucinatórios e delirantes associados ao uso agudo. A partir de 7,5 mg ao dia é que se observa algum efeito antiparkinsoniano, embora em nossa experiência, a maior parte dos pacientes responde bem com doses entre 10 e $30 \mathrm{mg}$ ao dia, podendo-se chegar a $60 \mathrm{mg}$ ao dia. Na fase inicial de adaptação à bromocriptina, necessita- 
se administrar o antagonista dopaminérgico periférico, domperidona para inibir os efeitos adversos. Na fase de uso crônico são descritos edema de extremidades e derrame pleural como efeitos diretamente relacionados.

O segundo derivado ergolínico, a pergolida, surgiu nos anos 1980 e foi lançada com a vantagem de ter um perfil de tolerabilidade um pouco melhor do que o da bromocriptina ${ }^{6}$. Atua estimulando os receptores D1, D2 e D3, embora seja mais bem tolerada, também necessita de uma titulação da dose de forma lenta. A dose inicial é de $0,05 \mathrm{mg}$ ao dia, com aumentos a cada 2 a 5 dias de 0,05 a 0,1 mg até atingir a dose de mínima de 0,25 mg três vezes ao dia. A partir dessa dose, faz-se incrementos semanais de 0,25 $\mathrm{mg}$ ao dia até que se obtenha o efeito desejado. Na média, há um efeito antiparkinsoniano satisfatório nas doses entre 1 e $3 \mathrm{mg}$ ao dia, podendo-se chegar a $6 \mathrm{mg}$ ao dia. Além dos efeitos adversos observados com a bromocriptina, recentemente foram descritas complicações relacionadas ao aparecimento de disfunções valvares cardíacas ${ }^{7,8}$.

A cabergolina é uma droga com uma boa ação dopaminérgica, com a vantagem de ter uma duração de efeito muito maior que os demais $A D$, pois tem uma meia vida entre 65 e 96 horas. Isso acaba permitindo que o paciente faça uma única tomada da medicação ao dia e permite que a estimulação dopaminérgica obtida seja mais próxima da fisiológica. O custo da medicação é elevado não sendo ainda acessível para a maioria das pessoas. Um bom efeito antiparkinsoniano é obtido com doses em torno de $2 \mathrm{mg}$ ao dia. Os principais efeitos adversos são sedação, edema de extremidades e náuseas. Também já foram descritos casos de fibrose valvar cardíaca associados à cabergolina ${ }^{7}$.

A lisurida é um AD que pode ser administrado por via oral ou subcutânea, tendo um perfil farmacológico semelhante ao da pergolida, com suas vantagens e efeitos colaterais próprios da classe.

\section{Agonistas não ergolínicos}

Dentre os $A D$ não ergolínicos, o mais antigo é a apomorfina, que vem sendo usada na Europa desde os anos 1950 para casos de intoxicação exógena por sua ação emética. No final dos anos 1960 sua ação AD foi ressaltada pelo mesmo grupo que fez os ensaios terapêuticos com a levodopa ${ }^{9}$. Entretanto, as dificuldades referentes à administração da substância pela via oral e a sua ação emética difícil de ser superada sem que houvesse piora dos sinais parkinsonianos, fez com que fosse colocada de lado até os anos 1980. Com o surgimento da domperidona, uma droga antagonista dopaminérgica periférica que impede os vômitos sem que haja uma piora motora, ressurgiu o interesse pela apomorfina na DP. A melhor ação antiparkinsoniana ocorre com a via subcutânea, na dose de 1 a 3 mg por aplicação. O efeito antiparkinsoniano ocorre entre 10 e 15 minutos depois da injeção e dura entre 40 e 60 minutos. Além de náuseas e vômitos aliviados pela domperidona, podem ocorrer bocejos, sonolência e confusão mental em alguns raros casos ${ }^{10}$. A infusão contínua subcutânea por bomba de infusão tem sido demonstrada como uma arma eficaz no controle das flutuações motoras, pois consegue diminuir significativamente os períodos "off" dos pacientes em fase avançada ${ }^{11}$. A apomorfina não está disponível no Brasil, mas pode ser obtida a partir de importadores independentes.

O piribedil é outro AD em uso desde o final dos anos 1970. Tem uma ação antiparkinsoniana discreta e é administrada por via oral nas doses 150 a 300 mg ao dia, dividida em 3 ou 4 tomadas. Atua sobre receptores D2 e D3 e sua maior limitação é a intensa ação nauseante e emética observada nos primeiros meses de uso. O uso da domperidona é praticamente obrigatório por 2 ou 3 meses de tratamento até que o paciente adaptese à droga. Alucinações, confusão mental e hipotensão arterial podem ser observadas em alguns pacientes. Tem a desvantagem de ter uma ação antiparkinsoniana discreta se comparada aos demais $A D$, mas a vantagem de ter um custo mais acessível.

O pramipexol é um AD lançado comercialmente no final dos anos 1990 com ação essencialmente sobre receptores D2 e D3 e tendo um perfil de tolerabilidade muito superior aos ergolínicos, ao piribedil e à apomorfina ${ }^{12}$. Tem uma potência farmacológica no mínimo igual a da bromocriptina e pergolida com a vantagem de ser bem tolerada e com um tempo de titulação não maior do que 3 semanas para se chegar à dose mínima efetiva. A meia vida plasmática é de 8 a 10 horas. Recomenda-se iniciar o pramipexol com doses de $0,125 \mathrm{mg}$, três vezes ao dia e dobrar a dose a cada 7 a 10 dias, até se atingir a dose mínima 
efetiva, que é de $0,5 \mathrm{mg} 3$ vezes ao dia. A dose útil da medicação gira entre $1,5 \mathrm{mg}$ a $4,5 \mathrm{mg}$ ao dia, dependendo de cada paciente. Algum grau de intolerância gastrintestinal pode ocorrer nos primeiros dias de uso, mas quase nunca requer o uso de antieméticos ou de suspensão da droga. Sonolência excessiva ou ataques de sono incoercíveis são observados em alguns pacientes e requer observação cuidadosa ou mesmo suspensão da medicação.

O ropinirol tem um perfil farmacológico semelhante ao do pramipexol sendo que a dose efetiva é de 8 a $18 \mathrm{mg}$ ao dia, mas, como no caso do pramipexol, tem que se iniciar com doses baixas, escalando-a semanalmente até que se atinja o objetivo ${ }^{13}$. O ropinirol não está ainda disponível nas farmácias brasileiras, embora possa ser adquirido através de importadores independentes.

\section{Uso de $A D$ nas fases iniciais da DP}

Os AD mostraram-se eficazes para a melhora dos sintomas parkinsonianos desde o surgimento da bromocriptina. Um estudo de 1993, com 782 pacientes mostrou que a bromocriptina usada precocemente na DP tendo a levodopa associada mais tardiamente foi capaz de diminuir a freqüência das oscilações motoras relacionadas à levodopa se comparada ao grupo que recebe levodopa logo no início da DP ${ }^{14}$. Com o surgimento dos novos $A D$ não ergolínicos, especialmente o ropinirol e o pramipexol, com um perfil de tolerabilidade superior aos outros $A D$, aumentou-se a experiência com o uso dessas drogas nas fases oligossintomáticas da doença. A despeito de serem eficazes nenhum dos $A D$ é tão potente quanto à levodopa no controle dos sintomas parkinsonianos ${ }^{12,13,15,16}$. Já foi demonstrado que a monoterapia com $A D$ pode ser mantida por um longo período de tempo. Um estudo com ropinirol demonstrou que $50 \%$ dos pacientes conseguem manter-se em monoterapia por até três anos e 30\% por 5 anos $^{13}$. Resultados similares são encontrados com o pramipexol.

O pramipexol em monoterapia ou associado a levodopa, em um estudo de dois anos, mostrou uma freqüência de complicações de longo prazo do tratamento da DP, como flutuações motoras e discinesias, da ordem de $28 \%$, contra $51 \%$ dos pacientes em uso de levodopa isolada ${ }^{17}$. Após quatro anos de uso contínuo a freqüência de discinesia é de 24,5\% para quem recebeu pramipexol como tratamento inicial e de 54\% para quem recebeu levodopa. Neste mesmo período de tempo o desenvolvimento de encurtamento do tempo de efeito da levodopa ("wearing off") ocorreu em $47 \%$ dos pacientes que iniciaram o tratamento com pramipexol e só depois tiveram a adição da levodopa ao tratamento contra $63 \%$ dos pacientes que já começaram o tratamento com a levodopa ${ }^{18}$.

\section{Uso dos $A D$ na fase avançada da DP}

Os $A D$ são drogas úteis no controle dos sintomas da fase avançada. Nos pacientes em uso de levodopa que desenvolvem flutuações motoras, como as oscilações em "on-off" e as discinesias costumam melhorar muito quando se adiciona $\mathrm{O} A D$ ao tratamento. $A$ adição do $A D$ permite que haja uma redução nas doses da levodopa sem que haja uma piora do desempenho motor. Este efeito positivo sobre as complicações de longo prazo da levodopa também parece ser exercido pela meia vida maior dos $A D$ quando comparada à da levodopa.

Qualquer um dos AD pode desempenhar este papel. Há estudos convincentes com a apomorfina por bomba de infusão contínua subcutânea na redução de períodos "off", nas flutuações randômicas do desempenho motor e nas discinesias ${ }^{19}$. A bromocriptina é o $A D$ há mais tempo em uso e há uma experiência significativa com a droga na fase avançada da DP 20. Entretanto, nos últimos anos, o uso do pramipexol vem superando de longe a bromocriptina devida à grande facilidade de uso e à menor incidência de efeitos colaterais. Um dos poucos estudos comparativos entre os $A D$ revelou que tanto o pramipexol (na dose de 4,5 mg ao dia) quanto a bromocriptina (na dose de $30 \mathrm{mg}$ ao dia) são eficazes no controle das complicações, com uma tendência de vantagem para o pramipexol ${ }^{21}$.

\section{Ação neuroprotetora dos AD}

Há estudos com PET scan utilizando fluorodopa ou com SPECT com radioligantes nos transportadores da dopamina do neurônio pré-sináptico que conseguem mensurar a perda neuronal no estriado do parkinsoniano. Um estudo comparando a perda neuronal nos pacientes com DP mostrou que, em 34 meses de acompanhamento com SPECT usando beta-CIT (um radioligante da proteína transportadora da dopamina), os pacientes usando levodopa têm um 
ritmo de perda neuronal de 19,6\% contra 10,9\% nos usando pramipexol ${ }^{22}$. A comparação da perda neuronal com levodopa e ropinirol foi feita utilizando-se o PET scan com fluorodopa. A perda neuronal comparando-se o exame do início do tratamento como de 24 meses depois mostrou que a perda neuronal foi de $20 \%$ no grupo que recebeu levodopa e de $13 \%$ no grupo que recebeu ropinirol ${ }^{23}$.

A relevância clínica desses achados ainda não está muito clara. De qualquer modo, há evidências de que o pramipexol e o ropinirol têm algum efeito poupador de neurônios. Há diversos mecanismos que poderíamos explicar esse suposto efeito neuroprotetor. Um deles é pelo efeito poupador de levodopa, ou seja, seria uma neuroproteção indireta já que nesta hipótese a levodopa seria tóxica. Esta hipótese é difícil de se sustentar, pois há fortes evidências que a levodopa não seja tóxica. Outra possibilidade é a de que o AD estimularia os receptores pré-sinápticos, reduzindo a síntese de dopamina, portanto diminuindo o metabolismo neuronal e a formação de radicais livres. Por último, há uma possibilidade que ocorra uma ativação de receptores com efeitos antiapoptóticos.

\section{REFERÊNCIAS BIBLIOGRÁFICAS}

1. Obeso JA, Rodriguez-Oroz MC, Rodriguez M, Macias R, Alvarez L, Guridi J, Vitek J, DeLong MR. Pathophysiologic basis of surgery for Parkinson's disease. Neurology. 2000;55(12 Suppl 6):S7-12.

2. Jenner P. Dopamine agonists, receptor selectivity and dyskinesia induction in Parkinson's disease. Curr Opin Neurol. 2003;16 (Suppl 1):S3-7.

3. Cotzias GC, Van Woert MH, Schiffer LM. Aromatic amino acids and modification of Parkinsonism. N Engl J Med 1967;276:374-379.

4. Jankovic J, Marsden CD. Therapeutic strategies in Parkinson's disease. In Jankovic J, Tolosa E, eds. Parkinson's disease and movement disorders, $3^{\text {rd }}$. edition. Williams \& Wilkins, Baltimore, 1998: 191-220.

5. Calne DB, Teychenne PF, Leigh PN, Bamji AN, Greenacre JK.. Treatment of parkinsonism with bromocriptine. Lancet. 1974;2:1355-1356.

6. Koller WC, Weiner WJ, Diamond BI, Nausieda PA, Klawans HL. The pharmacological evaluation of pergolide mesylate as a potential anti-parkinson agent. Neuropharmacology. 1980;19:831-837.

7. Horvath J, Fross RD, Kleiner-Fisman G, Lerch R, Stalder H, Liaudat S, et al. Severe multivalvar heart disease: a new complication of the ergot derivative dopamine agonist. Mov Disord 2004:656-662.

8. Van Camp G, Flamez A, Cosyns B, Weytjens C, Muyldermans L, Van Zandijcke M, et al. Treatment of Parkinson's disease with pergolide and relation to restrictive valvular heart disease. Lancet 2004; 363:1179-1183.

9. Duby SE, Cotzias GC, Papavasiliou PS, Laurence WH. Injected apomorphine and orally administered levodopa in parkinsonism. Arch Neurol 1972;27:474-480.

10. Ferraz HB, Azevedo-Silva SMC, Borges V, Rocha MSG, Andrade LAF Apomorfina. Uma alternativa no controle das flutuações motoras da doença de Parkinson. Arq Neuropsiquiatr 1995;53:245-251.

11. Frankel JP, Lees AJ, Kempster PA, Stern GM. Subcutaneous apomorphine in the treatment of Parkinson's disease. J Neurol Neurosurg Psychiatry 1990;53:96-101.

12. Shannon KM, Bennett JP Jr, Friedman JH. Efficacy of pramipexole, a novel dopamine agonist, as monotherapy in mild to moderate Parkinson's disease. The Pramipexole Study Group. Neurology 1997;49:724-728.

\section{Papel dos AD na doença de Parkinson}

Os $A D$ são drogas que podem ser utilizadas em qualquer fase da DP. Na experiência clínica do serviço de Distúrbios do Movimento da Disciplina de Neurologia da Universidade Federal de São Paulo, os AD são utilizados, quando possível, na fase inicial da DP, se os sintomas não são altamente incapacitantes e se o paciente tem abaixo de 70 anos de idade. Nos pacientes muito pouco sintomáticos, optamos por utilizar selegilina, amantadina ou anticolinérgicos. Nos pacientes com um alto grau de incapacitação ou se têm sintomas não tão incapacitantes, mas têm acima de 70 anos, damos preferência para a utilização da levodopa logo de início.

$\mathrm{Na}$ fase avançada da DP, quando ocorrem encurtamento do efeito ("wearing-off"), flutuações motoras ("on-off") e discinesias, os AD são indicados em associação à levodopa, por permitirem uma redução da doses dessa última, diminuindo as complicações. Nas fases muito avançadas, quase sempre utilizamos a combinação de levodopa, AD, inibidor da COMT e amantadina e, mesmo assim, nem sempre obtemos um sucesso terapêutico completo.
13. Rascol O, Brooks DJ, Korczyn AD, De Deyn PP, Clarke CE, Lang AE. A five year study of the incidence of dyskinesia in patients with early Parkinson's disease who were treated with ropinirole or levodopa. N Engl J Med 2000;342:1484-1491

14. Parkinson's Disease Research Group of the United Kingdom. Comparisons of therapeutic effects of levodopa, levodopa and selegiline, and bromocriptine in patients with early, mild Parkinson's disease: three years interim report. BMJ 1993; 307: 469_472

15. Uitti RJ, Ahlskog JE. Comparative review of dopamine receptor agonist in Parkinson's disease. Drugs 1996;5:369-388.

16. Hubble JP, Koller WC, Cutler NR, Sramek JJ, Friedman J, Goetz CG et al. Pramipexole in patients with early Parkinson's disease. Clin Neuropharmacol. 1995; 18:338-347.

17. Parkinson Study Group. Pramipexole vs levodopa as initial treatment for Parkinson's disease. JAMA 2000;284:1931-1938.

18. Holloway RG, Shoulson I, Fahn S, Kieburtz K, Lang AE, Marek K, et al. Pramipexole vs levodopa as initial treatment for Parkinson disease: a 4year randomized controlled trial. Arch Neurol 2004;61:1044-1053.

19. Colzi A, Turner K, Lees AJ. Continuous subcutaneous waking day apomorphine in the long term treatment of levodopa induced interdose dyskinesias in Parkinson's disease.

J Neurol Neurosurg Psychiatry. 1998:64:573-576.

20. Hoehn MM, Elton RL. Low dosage of bromocriptine added to levodopa in Parkinson's disease. Neurology 1985;35:199-206.

21. Guttman M. Double-blind comparison of pramipexole and bromocriptine treatment with placebo in advanced Parkinson's disease. International Pramipexole-Bromocriptine Study Group. Neurology 1997;49:1060-1065.

22. Parkinson Study Group. Dopamine transporter brain imaging to assess the effects of pramipexol vs levodopa on Parkinson's disease progression. JAMA 2002;287:1653-1661.

23. Whone A, Watts RL, Stoessl AJ, Davis M, Reske S, Nahmias C et al. Slower progression of Parkinson's disease with ropinirole versus levodopa: The REAL-PET study. Ann Neurol. 2003;54:93-101. 